\title{
Flexible Multi-Band OFDM Receiver Based on Optical Down- Conversion for Millimeter Waveband Wireless Base Stations
}

\author{
P. Ghelfi $i^{(1)}$, G. Serafino ${ }^{(2)}, F$. Scotti $^{(1)}, F$. Laghezza ${ }^{(3)}$, A. Bogoni $^{(1)}$ \\ (1) CNIT - National Laboratory of Photonic Networks, Pisa, Italy, paolo.ghelfi@cnit.it \\ (2) Scuola Superiore Sant'Anna, Pisa, Italy \\ (3) CNIT - National Laboratory of Radar and Surveillance Systems, Pisa, Italy
}

\begin{abstract}
A$ novel and flexible photonics-based down-conversion scheme is proposed for wireless receivers in base stations. It allows simultaneous detection of multiple signals at carriers up to tens of $\mathrm{GHz}$, enabling communications at millimeter waves. Experiments demonstrate the effective downconversion of Wi-Fi signals at 2.4 and 39.8GHz with EVM<-43dB.
\end{abstract}

\section{Introduction}

The growing demand for mobile broadband services is putting wireless networks under tremendous pressure. The capacity increase of new protocols as LTE (Long Term Evolution) and LTE-Advanced, and the reduction of network cell dimensions, will be inadequate to address all the future bandwidth requirements ${ }^{1}$. The wireless community is therefore considering to extend the exploited spectrum to the millimeter waveband (MMB, $3-300 \mathrm{GHz}$ ) which is underutilized and offers enormous bandwidth ${ }^{2}$. Orthogonal frequency division multiplexing (OFDM) will probably be the preferred airinterface due to its resilience and flexibility, but its detection requires local oscillators with good phase stability to avoid inter-channel interference. In today's base stations, the wireless equipments cannot simultaneously manage wireless signals ranging from few $\mathrm{MHz}$ to several $\mathrm{GHz}$. If a single transceiver with the capability of treating multiple frequency bands up to the MMB were available, it would strongly reduce the hardware requirements, and will enable the implementation of a flexible multiprotocol base station for future mobile networks. To the best of our knowledge, only one paper has explored the down-conversion of multiple signals ${ }^{3}$, but applying a statistical approach that does not fit the mobile communication scenario.
In this paper we propose a novel flexible wireless receiver that exploits a mode locked laser (MLL) to simultaneously and precisely down-convert signals from several different bands ranging up to the $\mathrm{MMB}$, without requiring frequency- or protocol-specific RF components. This way the huge data traffic managed by the base station can be received and processed at low frequency where detection is cheaper. We believe this receiver scheme allows the realization of future-proof mobile systems.

\section{Concept}

The proposed optical down-converter is based on the concept of optical sampling (see Fig. 1 for reference). If an optical pulse train (e.g., a $M L L)$ with repetition rate $f_{\text {Rep }}$ is modulated by an RF signal at $f_{R F}$, the spectrum of the modulating signal is replicated every $f_{\text {Rep }}$ on the spectrum. When the modulated optical signal is revealed in a photodiode (PD), the heterodyne process will generate a copy of the RF signal downconverted within the range from DC to $f_{\text {Rep. }}$. If the pulse train is modulated by the sum of several RF signals at different frequencies, all the RF signals will be down-converted to below $f_{\text {Rep }}$ and, provided the pulse repetition rate is correctly chosen, they will not overlap with each other. Since the electrical spectrum of interest is limited to $f_{\text {Rep }}$, the required PD can have an E/O

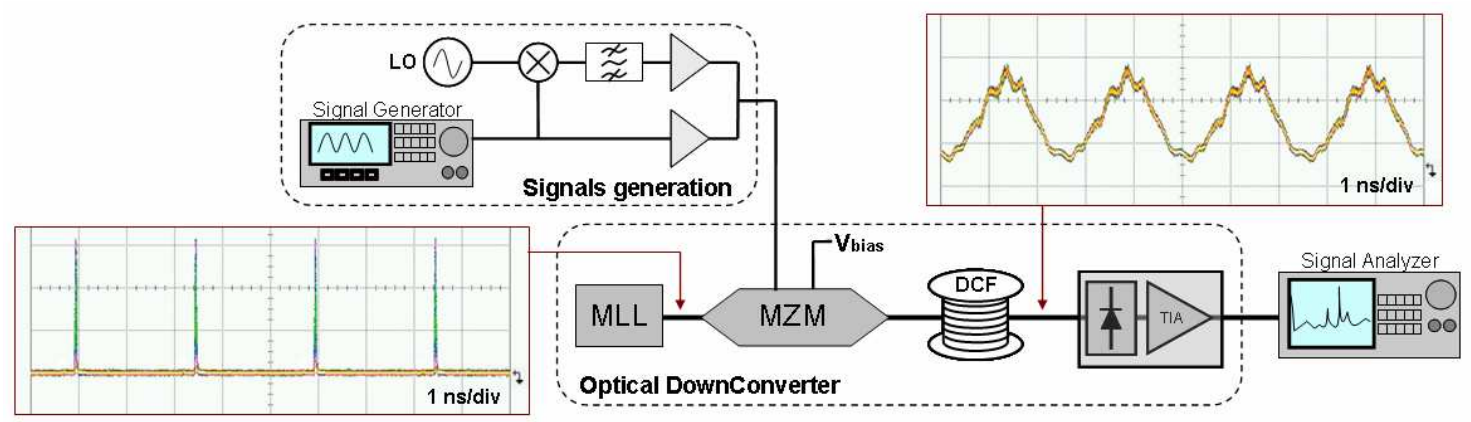

Fig. 1: Experimental setup. LO: Local Oscillator; MLL: Mode-Locked Laser; MZM: Mach-Zehnder Modulator; DCF: Dispersion Compensating Fiber; TIA: Trans-Impedance Amplifier. Insets: waveforms of the pulses before and after the DCF, as seen by the exploited photodiode (bandwidth $10 \mathrm{GHz}$ ). 
bandwidth just larger than $f_{\text {Rep }}$. On the other hand, the bandwidth of the pulsed laser must be larger than the maximum RF frequency to be down-converted. In order to match the bandwidth of the detected pulses to the analysis range, it is convenient to broaden the pulses (e.g., through chromatic dispersion) so that most of the power detected by the PD is below $f_{\text {Rep }}$.

\section{Experiment}

The experimental setup is depicted in Fig. 1. A multi-band RF signal is first generated to test the proposed receiver. A vector signal generator (Agilent MXG-N5182) is exploited to generate 52 OFDM sub-carriers around $2.425 \mathrm{GHz}$, carrying 54Mbps over a 64-QAM pattern, according to the $802.11 \mathrm{~g}$ standard. To obtain two signal bands, the Wi-Fi signal is split and up-converted to $39.825 \mathrm{GHz}$ via an $\mathrm{RF}$ mixer driven by a local oscillator at $37.4 \mathrm{GHz}$. A bandpass filter is used for rejecting the image frequency before summing the two signal bands together. A delay is also inserted for decorrelating the waveforms. The spectrum of the dual-band signal thus generated is reported in Fig. 2. The insets show the two Wi-Fi frames. The signal at $39.825 \mathrm{GHz}$ suffers non-uniform channel power due to the narrow bandwidth $(40 \mathrm{MHz}$ at $39.812 \mathrm{GHz})$ of the exploited RF filter.

In the optical down-converter, the generated

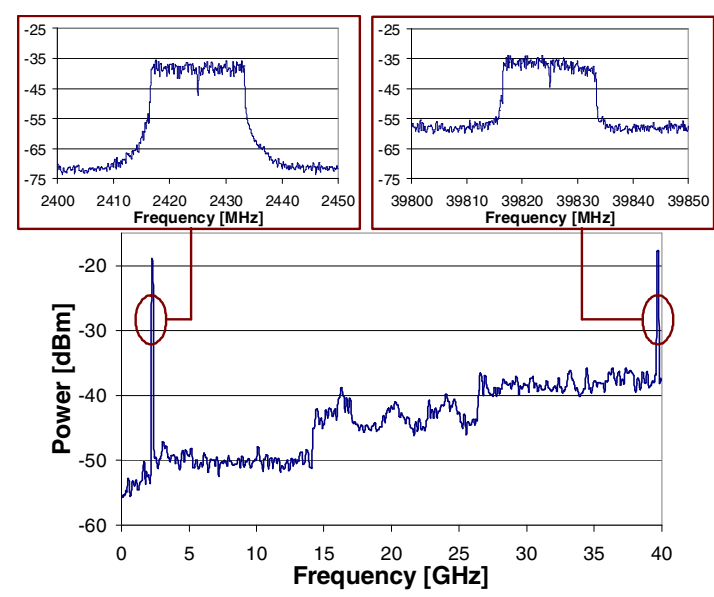

Fig. 2: Electrical spectrum of the multi-band RF signal. Insets: OFDM spectra at $2.425 \mathrm{GHz}$ (right) and at $39.825 \mathrm{GHz}$ (left).

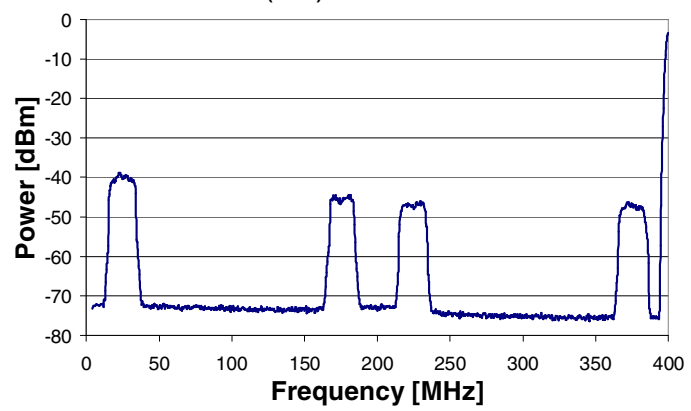

Fig. 3: Down-converted signals from $D C$ to $400 \mathrm{MHz}$. multi-band signal modulates a pulsed laser in a Mach-Zehnder modulator (MZM) driven in its linear region. The laser is a passive MLL at $1554 \mathrm{~nm}$ with a repetition frequency of $400 \mathrm{MHz}$ and a pulsewidth of $1.5 \mathrm{ps}$. A spool of dispersion compensating fiber (DCF) with a total dispersion of $320 \mathrm{ps} / \mathrm{nm}$ is inserted to broaden the pulses, so that they resemble a sinus wave at $400 \mathrm{MHz}$ (see inset in Fig. 1). The optical signal is then detected by a photodiode (PD), thus realizing the down-conversion of the two RF signals simultaneously. The electrical spectrum generated by the PD between DC and $400 \mathrm{MHz}$ is reported in Fig. 3. The signal at $2.425 \mathrm{GHz}$ is down-converted at $25 \mathrm{MHz}$, and the signal at $39.825 \mathrm{GHz}$ is moved at $225 \mathrm{MHz}$. The respective image-frequency signals at $375 \mathrm{MHz}$ and $175 \mathrm{MHz}$ are also generated. The first harmonic of the laser pulses is also visible at $400 \mathrm{MHz}$. Finally the signal replicas at $25 \mathrm{MHz}$ and $225 \mathrm{MHz}$ are received by a vector signal analyzer (Agilent MXG).

Fig. 4a) reports the Error Vector Magnitude (EVM) measured on the down-converted $2.425 \mathrm{GHz}$ signal varying the RF power at the input of the MZM (squares), while maintaining the $39.825 \mathrm{GHz}$ channel at its optimum power $(-4 \mathrm{dBm})$. Increasing the RF input power, the EVM decreases due to the improving signal-tonoise ratio, until a minimum EVM of $-43 \mathrm{~dB}$ is reached at $-2 \mathrm{dBm}$. From this point on, after a short region of stable behaviour, the EVM starts increasing due to the saturation of the RF amplifiers used in the signal generation. The saturation cannot be ascribed to the transfer function of the MZM, since the total RF input power is low $\left(<5 \mathrm{dBm}\right.$, while the MZM $V_{\pi}$ is about $6 \mathrm{~V})$. The excellent quality of the down-converted signal is demonstrated by the inset in Fig. 4a) showing the demodulated 64-QAM constellation for the lowest EVM. The analysis has been repeated switching off the signal at $39.825 \mathrm{GHz}$ (diamonds), obtaining almost identical results and demonstrating that the simultaneous downconversion of more than one channel does not affect the performance of the system. The results are compared with the direct demodulation of the $2.425 \mathrm{GHz}$ signal at the vector signal analyzer (triangles). The back-toback curve reaches the minimum EVM of $-44.7 \mathrm{~dB}$ at $-14 \mathrm{dBm}$, and keeps constant to about OdBM where the saturation of the RF amplifiers makes it increasing. The comparison between the back-to-back and the optically downconverted signals reveals that the proposed scheme shows only a small performance reduction, due to the out-of-band noise downconverted to the analysis range, and a decrease 
of sensitivity related to the $V_{\pi}$ of the used MZM.

The analysis has been repeated on the signal at $39.825 \mathrm{GHz}$, as reported in Fig. 4b). The curve related to the down-converted signal at $39.825 \mathrm{GHz}$ taken with the $2.425 \mathrm{GHz}$ signal at its optimum power $(-2 \mathrm{dBm})$ (squares) shows the same behaviour as the previous case, except for the minimum EVM that here is $-34.5 \mathrm{~dB}$ due to the quality of the generated signal. Nevertheless the constellation reported in the inset for the best EVM is very clear. With respect to the curve obtained switching off the signal at 2.425GHz (diamonds), the measures show an EVM penalty of $0.5 \mathrm{~dB}$ at low input power that disappears when the minimum EVM is reached. This degradation can be ascribed to a lack of isolation in the signal generation scheme, which allows the signal at $2.425 \mathrm{GHz}$ to counterpropagate in the $39.825 \mathrm{GHz}$ amplifier reducing its efficiency. Since the vector signal analyzer cannot directly demodulate the signal at $39.825 \mathrm{GHz}$, here the optical down-conversion has been compared to an RF down-conversion from $39.825 \mathrm{GHz}$ to $2.425 \mathrm{GHz}$ (triangles). The back-to-back curve reaches the EVM floor at $-13 \mathrm{dBm}$, with a performance only $1.7 \mathrm{~dB}$ better.

\section{Comments and conclusions}

The experimental results confirm that the proposed optical down-converter can simultaneously deal with multiple RF signals over a very wide frequency range, and without frequency-dependent performance degradations that are typical of RF down-conversions. This is due to the fact that common MLLs show a better
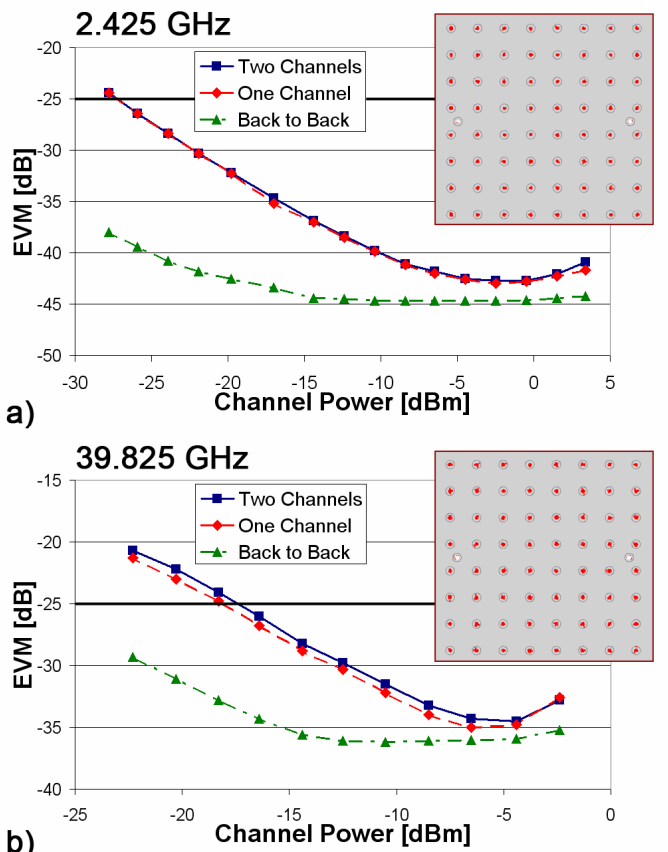

Fig. 4: EVM versus modulating signal power at a) $2.425 \mathrm{GHz}$ and b) $39.825 \mathrm{GHz}$ bands. Insets: Relative constellations at minimum EVM. phase stability than high frequency oscillators, thus allowing a precise down-conversion of OFDM frames even at very high frequency, as demonstrated by our experiment. Integrated MLLs also shows a significant tunability in repetition rate which can help reconfiguring the optical down-converter. The small penalty measured in the EVM, due to the folded out-ofband noise, can be reduced by a fine design of the amplifiers bandwidths. The bandwidth limit of the proposed scheme is set by the electrooptical bandwidth of the modulator, which anyway can reach $110 \mathrm{GHz}^{4}$. A dual parallel MZM can also be used to realize a single-side band optical modulation, thus avoiding the image-frequency components in the downconverted frequency range, and eliminating the risk for dispersion effects ${ }^{5}$. Finally, an improve in the down-converter sensitivity can be achieved with modulators with reduced $\mathrm{V}_{\pi}$.

In conclusion, we have proposed and demonstrated a novel optical down-converter scheme that exploits a MLL to simultaneously and precisely down-convert RF signals from several different bands, ranging up to the MMB. The scheme is suitable for the use in future wireless base stations managing large amount of data over several different protocols and carrier frequencies, since it can flexibly and precisely down-convert any RF signal without requiring frequency- or protocol-specific $R F$ components. This feature also allows the reconfigurability of the base station to new transmission protocols without changing the hardware, thus reducing the capital expenditure. Finally, we believe this architecture can help the realization of future-proof flexible mobile systems.

\section{Acknowledgements}

This work has been supported by the ERC project PHODIR and the Italian Defence Ministry project SOPHIA.

The authors thank Dr. M. Presi and Dr. G. Cossu for fruitful collaborations.

\section{References}

[1] White_paper_c11-520862, February 2011.

[2] WiGig White Paper

[3] A. Feldster et al., J. Lightwave Technol., 27, 8 (2009).

[4] D. Chen et al., Appl. Phys. Lett., 70, 3335 (1997)

[5] G.H. Smith et al., Trans. Microw. Theory Techn., 45, 8 (1997) 